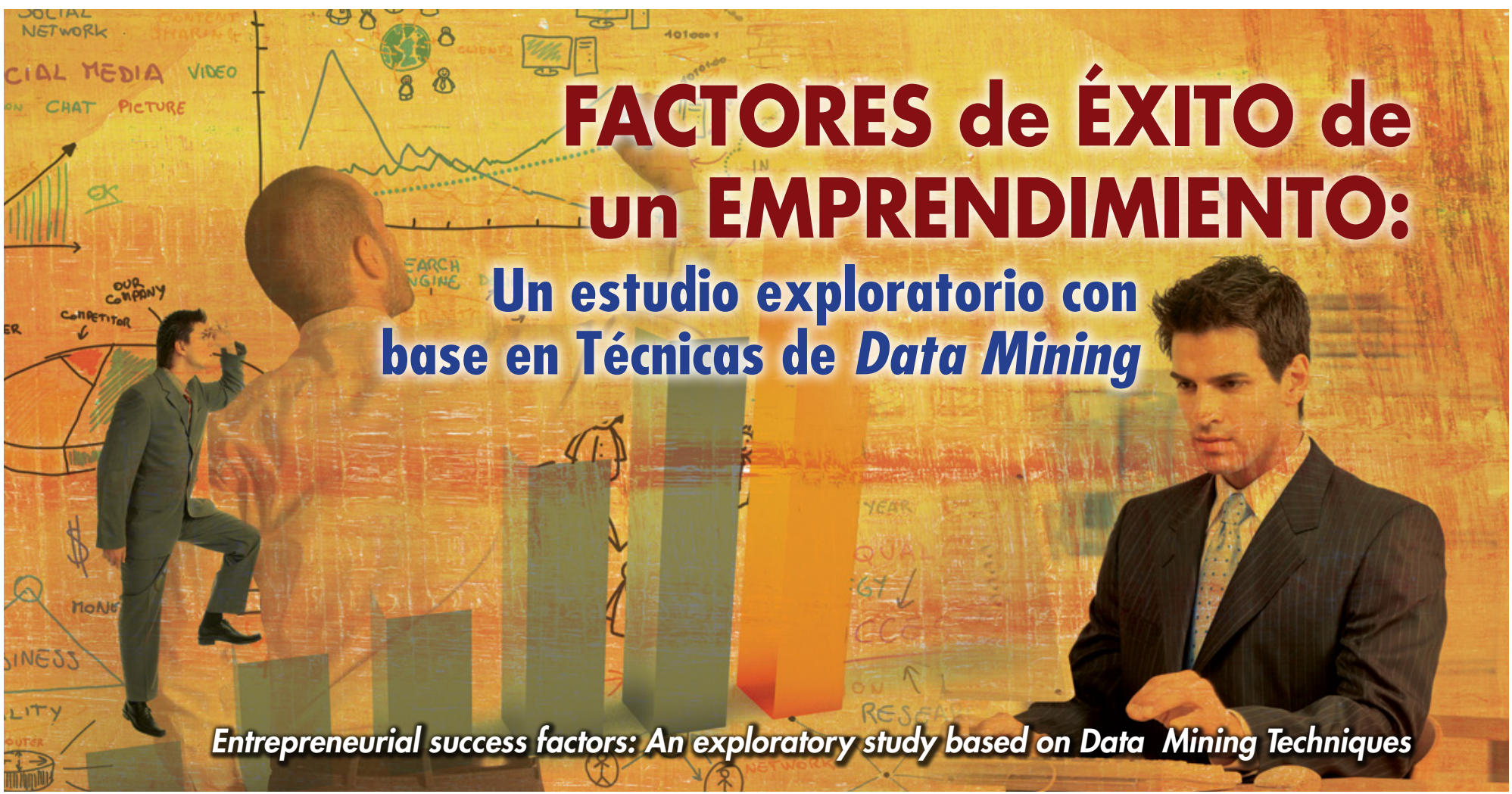

Tec Empresarial, Abril - Julio 2015, Vol 9 Núm 1 / p. 31-40

\section{María Messina}

messina@ccee.edu.vy

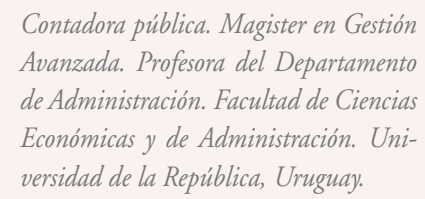

Contadora pública. Magister en Gestión Avanzada. Profesora del Departamento de Administración. Facultad de Ciencias Económicas y de Administración. Universidad de la Repuiblica, Uruguay.

\section{Esther Hochsztain esther@ccee.edu.uy}

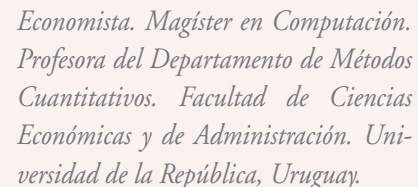

- Recepción del artículo: 9 junio, 2014

- Aprobación del artículo: 17 febrero, 2015

\title{
ABSTRACT
}

Since 2007, the CCEE Entrepreneurship Centre has developed a supporting program for entrepreneurs. A preliminary analysis to determine if the venture was successful or a failure is made to improve the program's management .

In this article, the authors identify the main factors associated with entrepreneurship's success, and how they can anticipate entrepreneurship's performance.

The case study is based on a survey data applied to the Entrepreneurship Program participants. The two Keywords: Entrepreneurship, entrepreneurial context, data mining, entrepreneurial success, entrepreneurship process.

\section{RESUMEN}

El Centro de Emprendedurismo CCEEmprende desarrolla, desde 2007, un programa de apoyo a emprendedores. Para mejorar su gestión, resulta de gran importancia analizar, en forma preliminar, los emprendimientos en una de dos categorías: éxito o fracaso.

En este artículo se identifican los principales factores asociados al éxito de un emprendimiento y cómo se vinculan para anticipar el futuro del emprendimiento.

Se presenta un caso de estudio con base en los datos de una encuesta realizada a emprendedores participantes del programa, aplicando técnicas de clasificación. Las dos técnicas utilizadas de data mining son árbol de decisión y regresión logística, en ambas se obtuvieron resultados coin- data mining techniques are decision trees and logistic regression. The results were consistent across both techniques. The findings show that the two most important elements to predict entrepreneurship's success are funding and previous experience as self-employed.

The results provided very useful insight about the best ways to support entrepreneurship, how to encourage entrepreneurs, and define tools or activities to impact positively ventures success in Uruguay, since similar studies have not been developed.
Palabras clave: Emprendedurismo, contexto emprendedor, minería de datos, éxito emprendedor, proceso $\gg$ emprendedor cidentes. Los hallazgos muestran que los dos elementos más relevantes para anticipar el éxito de un emprendimiento son contar con financiamiento y que, anteriormente, la situación laboral del emprendedor sea trabajador independiente.

Estos primeros resultados obtenidos en el estudio de caso revelan información útil acerca de las mejores formas prendedor y la definición de herramientas o actividades que incidan favorablemente en el éxito de los emprendimientos. Si bien desde la teoría o para otras realidades existe información sobre los factores que colaboran en la determinación del éxito, para la realidad del Uruguay no se identifican estudios similares. de apoyo al emprendedor, cómo generar incentivos al em- 


\section{〉 INTRODUCCIÓN}

El emprendedurismo es considerado cada vez un factor más importante para contribuir al desarrollo económico, la generación de puestos de trabajo, la inclusión social, la reducción de la pobreza y el desarrollo de la innovación. Eso ha llevado al interés de políticos, académicos y organismos internacionales en el proceso de creación de nuevos emprendimientos. La Organización de Naciones Unidas (ONU) aprobó por unanimidad la resolución 67/2012 "Emprendimiento para el desarrollo", que reconoce formalmente que el emprendimiento es una herramienta importante para la reducción de la pobreza, la creación del desarrollo sustentable y la revitalización del entorno.

La educación universitaria cumple un rol fundamental en el proceso de desarrollo emprendedor. La universidad pasó de ser una institución de conservación, preservación y transmisión cultural y del conocimiento -tarea que mantuvo por más de seis siglos-a una institución que incorporó como propósito promover la transferencia de los resultados de la investigación a productos y nuevas empresas. La Declaración Mundial sobre Educación Superior de 1998, recogiendo el debate que se venía dando, establece en el artículo 7, inciso d) la importancia de aprender a emprender y fomentar el espíritu de iniciativa en la educación superior. En especial, teniendo en cuenta que los egresados deberán crear puestos de trabajo, y no solamente buscar su inserción laboral en las alternativas existentes.

En este artículo se presenta un caso de estudio con base en datos de emprendedores participantes del programa CCEEmprende, que brinda apoyo a emprendedores en el ámbito de la Facultad de Ciencias Económicas y de Administración de la Universidad de la República, en Uruguay. Se analiza, mediante la aplicación de dos técnicas de data mining, los principales factores asociados al éxito de un emprendimiento y la forma en que se vinculan para anticipar el futuro del emprendimiento. Asimismo, el artículo se estructura de la siguiente forma: en la segunda parte se presenta la situación de apoyo al emprendedurismo en el país; en el tercer capítulo se expone el marco teórico; en el cuarto se presenta el estudio del caso; para finalizar, en la quinta sección aparecen conclusiones y sugerencias para trabajos futuros.

\section{SITUACIÓN DEL APOYO AL EMPRENDEDURISMO EN URUGUAY}

A continuación se reseñan el Programa Emprender y el Programa de Apoyo a Futuros Empresarios (PAFE), que han dado marco al ecosistema emprendedor del Uruguay. Se presenta también el Programa de Emprendedores (CCEEmprende) de la Facultad de Ciencias Económicas y de Administración de la Universidad de la República. En el marco de CCEEmprende, se desarrolla este trabajo de investigación.

\section{Programa Emprender}

En 2007, el Banco Interamericano de Desarrollo, como administrador del Fondo Multilateral de Inversiones (FOMIN), suscribió un convenio de cooperación técnica no reembolsable con la Corporación Nacional para el Desarrollo (CND) y el Laboratorio Tecnológico del Uruguay (LATU), titulado Apoyo Integral a la Empresarialidad Dinámica, conocido como Programa Emprender. El objetivo del Programa Emprender fue "promover una cultura emprendedora de alto valor agregado que contribuya a la creación de valor y crecimiento sostenido de la economía uruguaya”.
Para el Programa Emprender, el LATU y la CND definieron:

- Visión: Ser promotor del emprendedurismo dinámico y que contribuya al crecimiento sostenido de la economía.

- Misión: Desarrollar una red de instituciones de apoyo al emprendedurismo a fin de lograr un mayor acercamiento del emprendedor, y su orientación a través de ella de manera integral para constituir empresas dinámicas.

- Estrategia: Generar incentivos para todos sus participantes de forma sostenible.

El Programa Emprender se llevó a cabo a través de la construcción de un ecosistema emprendedor, de un fondo de capital semilla y ángeles inversores. Se quería promover la creación, formación, fortalecimiento y financiamiento de emprendimientos dinámicos en el país. La conformación del ecosistema emprendedor se desarrolló a través de la participación de instituciones colaboradoras, con un modelo de gestión que buscó dar participación a instituciones y programas de apoyo emprendedor existentes y llenar vacíos que se encontraron a lo largo del proceso emprendedor.

\section{Programa PAFE}

El Programa Emprender, que finalizó a mediados de 2012, fue continuado por el Programa de Apoyo a Futuros Empresarios (PAFE), de la Agencia Nacional de Investigación e Innovación (ANII). De esa forma se buscó la continuidad del Ecosistema Emprendedor, denominado Red Emprender. El PAFE tiene como objetivo general contribuir a incrementar la inversión privada en actividades de innovación. De este programa depende la Red de Apoyo a Futuros Empresarios (RAFE), que está integrada por diferentes organizaciones públicas y privadas, empresas,

\section{El artículo identifica los principales factores asociados al éxito de un emprendimiento, basándose en una encuesta realizada a emprendedores participantes de un programa de apoyo en la república de Uruguay}


universidades, fundaciones, ONG, cámaras, inversionistas, etc.

RAFE apoya emprendimientos que comercialicen productos o servicios que se inserten en cadenas de valor, con un valor agregado diferencial. El apoyo es a lo largo de todo el proceso: desde la sensibilización hasta la inversión privada de nuevos emprendimientos. Los principales cometidos del programa son:

- Apoyo a la cultura emprendedora: acciones de sensibilización, preincubación, diseño y gestación de negocios.

- Apoyo al desarrollo inicial de emprendimientos: incubación, emprendedores del exterior, apoyar a entidades dedicadas a financiar etapas tempranas de nuevos emprendimientos así como redes de inversionistas privados.

\section{Programa CCEEmprende}

En el 2006, la Facultad de Ciencias Económicas y de Administración de la Universidad de la República comenzó a trabajar en el diseño de actividades destinadas a apoyar el desarrollo emprendedor. Esas actividades se ejecutaron a partir de 2007 a través del programa CCEEmprende.

Por medio de CCEEmprende, dicha facultad, desde 2007, ha sido una de las instituciones colaboradoras del Programa Emprender, el cual se orientó a trabajar con emprendimientos dinámicos y definidos como:

Aquella nueva o reciente iniciativa empresarial con alto potencial de crecimiento y horizonte exportador que genere valor agregado y logre diferenciarse a través de un modelo de negocio, producto o servicio innovador (por su diseño, proceso o tecnología aplicada), como para al menos convertirse en una mediana empresa ${ }^{1}$.

Si consideramos que el proceso emprendedor comprende las etapas de diseño y gestación, puesta en marcha y desarrollo inicial, CCEEmprende se ubica fundamentalmente

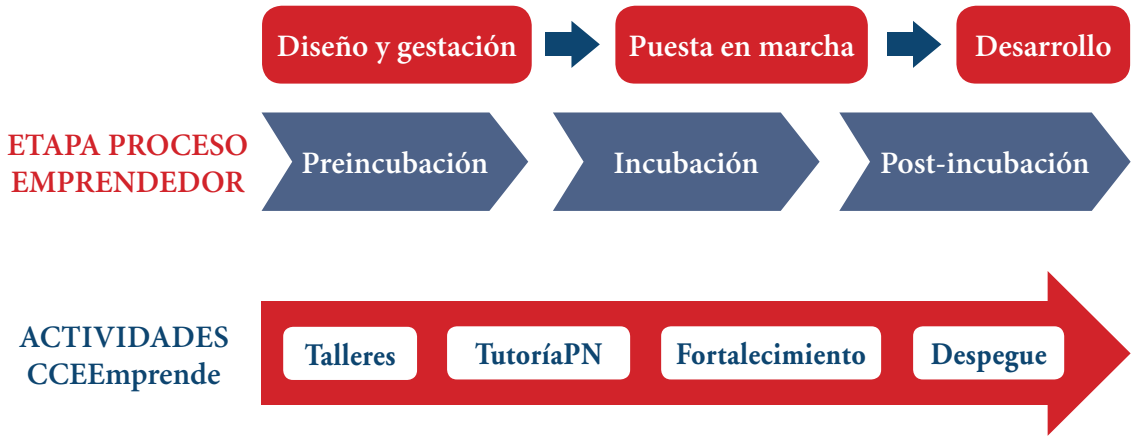

Figura 1: CCEEmprende en el proceso emprendedor

Fuente: Elaboración propia.

en la etapa de gestación, al dar asesoría para la puesta en marcha y comenzar a trabajar en el apoyo al desarrollo inicial. Si se consideran las etapas en las cuales se puede apoyar un emprendimiento, CCEEmprende se ubica en la etapa de pre-incubación, y comienza a desarrollar actividades de incubación (ver figura 1).

La misión definida en el 2007 para el programa CCEEmprende fue apoyar la creación y la gestión de emprendimientos dinámicos. También se pretende consolidar un equipo académico especializado en emprendimientos dinámicos que permita la actualización continua de conocimientos. En 2010 se formalizó el Programa CCEEmprende a través de la creación del Centro de Emprendedurismo.

En este trabajo se considera sólo la población de emprendedores que asistieron a los talleres de capacitación y terminaron el plan de negocios. Es relevante aclarar que los talleres y tutoría son abiertos a cualquier emprendimiento que sea potencialmente dinámico, sin ser un requisito ser emprendedor universitario (estudiante, docente o egresado de la universidad). A continuación se presentan los antecedentes teóricos relacionados con esta investigación.

\section{REVISIÓN TEÓRICA}

Diversos estudios estadísticos revelan que las nuevas empresas tienen verdaderas dificultades para sobrevivir, pues existe una "alta tasa" de mortandad que se presenta en los pri- meros cuatro o cinco años de vida (De Castro, Pistrui, Corduras, Cohen y Justo, 2002; Talín y Cuesta, 2013).

Para poder bajar esta tasa, es relevante conocer cuáles factores afectan la supervivencia. En ese sentido, debe tenerse presente que el surgimiento y el desarrollo de las empresas son fenómenos complejos y multidimensionales, además son considerados de naturaleza sistémica (Kantis, Federico y Menéndez, 2012).

Debido a la multiplicidad de factores que inciden sobre el emprendedor y el proceso emprendedor, se hace necesario para los países e instituciones derribar barreras inhibitorias del emprendimiento, actuando sobre la existencia de fallas sistémicas, es decir, limitaciones en el desarrollo de alguno de los factores que influyen sobre el proceso emprendedor (trámites y registros de empresas, acceso al financiamiento, peso de impuestos y regulaciones, infraestructura para la innovación, redes de contactos, entre otras) (Kantis, Angelelli y Moori-koenig, 2012).

A continuación se presentan los diferentes factores que inciden en el desarrollo emprendedor y su análisis empírico.

\section{Factores de éxito}

Para Gonzalo, Federico y Kantis (2013), el conjunto de características relevantes son: el perfil, background y las redes de los emprendedores; su acceso a recursos claves (financieros, humanos, información, etc.); el de- $\gg$ 
$\gg$ sarrollo de rutinas organizacionales; y, finalmente, el proceso de toma de decisiones. Otros autores hacen referencia a las características del emprendedor, el acceso a recursos y el tipo de proyecto dentro de los principales determinantes del crecimiento inicial de las empresas (Davidsson, Delmar y Wiklund, 2006).

Algunos autores hablan de factores socio-demográficos, que son aquellos ajenos al emprendedor, sobre los cuales no puede decidir. Estos factores son: sexo, edad y contexto (cultura, valores, normas formales e informales, instituciones y otros) (Leiva, 2013).

Fernández, Montes y Vázquez (2010) hacen referencia a la importancia de los recursos intangibles para el crecimiento empresarial. Por su parte, Capelleras y Kantis (2009) indican que, en general, el crecimiento y desarrollo de las nuevas empresas depende de varios factores que están vinculados con las características del emprendedor, las dimensiones del capital relacional y las características del mercado, junto con algunos elementos de tipo estratégico.

En esta investigación se seleccionaron los factores identificados por diferentes autores, algunos ya mencionados y otros que se presentarán a continuación. Los cuales pueden clasificarse en aquellos que hacen referencia a las características de los emprendedores y otros a las estrategias seguidas por la empresa.

\section{Características de los emprendedores}

Según lo comprobado por diferentes estudios, la edad del empresario en el momento de la creación de la empresa tiene un efecto negativo sobre el desarrollo temprano del nuevo negocio. La evidencia empírica ha demostrado que los empresarios más jóvenes son más ambiciosos, creativos y tienen más energía, lo que les permite superar su falta de credibilidad, de experiencia y la dificultad para conseguir financiamiento (Brüderl y Preisendörfer, 2000; Alemany, Álvarez, Planellas y Urbano, 2011). Los empresarios más viejos son generalmente más cautelosos y conservadores (Lafuente, Vaillant y Gómez, 2011). Otros autores señalan que la probabilidad de

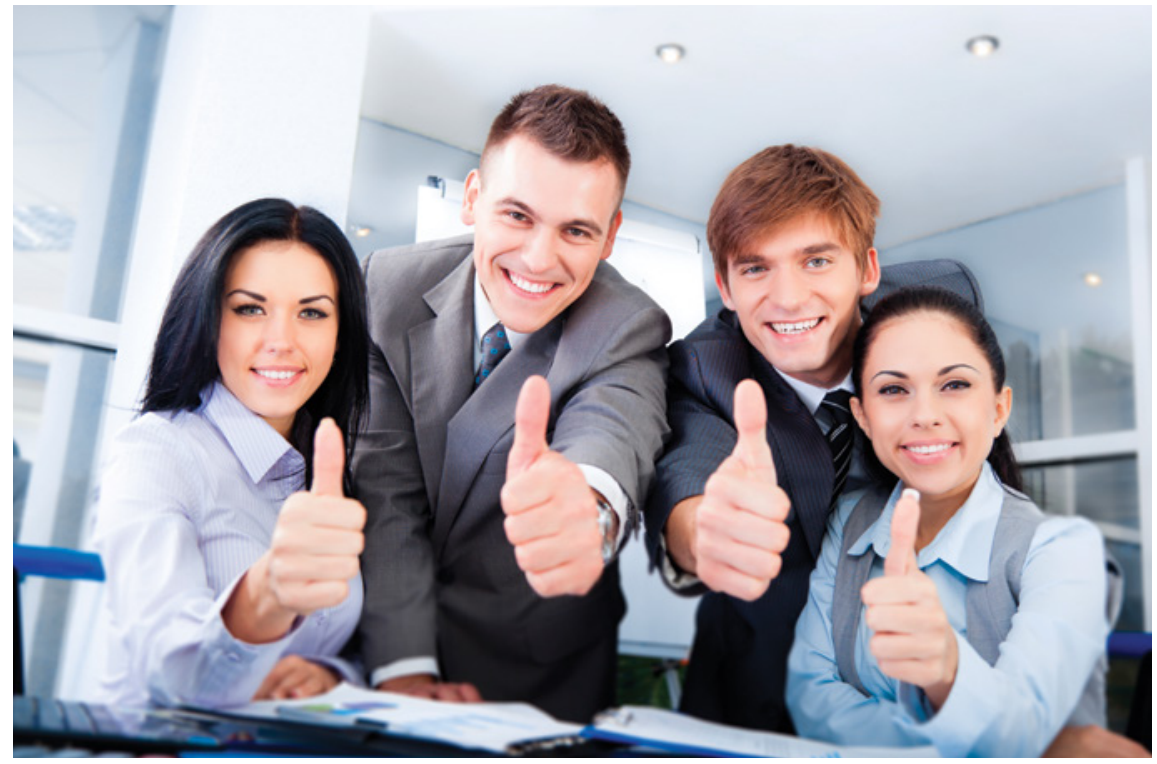

emprender en personas que tienen entre $18 \mathrm{y}$ 64 años decrece a medida que la edad del individuo aumenta (Vaillant y Lafuente, 2007).

Dentro de las variables socio-demográficas analizadas por varios autores en estudios empíricos, el sexo es relevante. Arribas y Vila (2004) comprobaron que el $44 \%$ de los hombres tiene la iniciativa de crear una empresa contra el 30,9\% de las mujeres.

Hay un importante número de estudios empíricos que demuestran el impacto positivo del perfil y del nivel de capital humano del fundador (o fundadores) sobre el crecimiento empresarial (Colombo y Grilli, 2005). Además, el capital humano comprende el conocimiento, las habilidades y las capacidades del emprendedor o equipo emprendedor, las cuales son consecuencia del nivel educativo y de la experiencia previa (Honig, 2001).

Está ampliamente reconocido que un nivel educativo alto incrementa las oportunidades de crecimiento empresarial. Podría considerarse que los empresarios más educados tienen las habilidades que les permite identificar y explotar las nuevas oportunidades de negocio (Alemany et al., 2011). También, la educación proporciona los conocimientos que pueden ayudar a superar las dificultades financieras (Evans y Leighton, 1989). Otros estudios demuestran que aquellas empresas que tienen tasas más altas del crecimiento del empleo tienen fundadores con estudios universitarios (Westhead y Cowling, 1995; Almus, 2002).

El otro componente del capital humano es la experiencia previa. Según García y Jiménez (2012), la experiencia laboral previa puede afectar el crecimiento empresarial. Particularmente, la experiencia previa como directivo y como emprendedor. Para Capelleres y Kantis (2009), la experiencia previa del empresario influye en el tiempo empleado para el proceso de creación de una nueva empresa. Otro factor analizado como determinante del éxito de las empresas es la motivación que lleva a emprender (Sastre, 2013). En general, los individuos que emprenden motivados por una situación de desempleo podrían no tener suficientes habilidades, preparación o experiencia para crear y desarrollar un emprendimiento.

Una corriente de investigación que ha adquirido mucha relevancia es la interesada en analizar cómo el conjunto de redes y el capital relacional del emprendedor inciden de manera determinante en la creación y en el éxito de nuevas empresas (Kantis et al., 2004; Capelleras y Greene, 2008; Capelleras y Kantis, 2009).

A través de sus redes, los empresarios pueden tener acceso a recursos, información y conocimientos muy importantes. Las redes son recursos valiosos y pueden compensar déficits 
de otros recursos, como el capital financiero y el capital humano (Gonzalo, Federico y Kantis, 2013)

El apoyo recibido de las redes facilita tanto la rápida gestación de la nueva empresa como su posterior crecimiento. Según Capelleres y Kantis (2009), en los casos en que el emprendedor tiene una red informal fuerte, el proceso de creación de la empresa se da más rápidamente que cuando el futuro empresario se apoya en los distintos tipos de programas de ayuda para la creación de su empresa (redes formales).

\section{Estrategias de la empresa}

Hasta el momento se han planteado factores que hacen referencia a las características de los emprendedores. A continuación se recogen estudios que identifican las estrategias tomadas por la empresa como factores de éxito.

Las estrategias de financiación, fundamentalmente las fuentes de financiación utilizadas, contribuyen a explicar la puesta en marcha y el crecimiento de las empresas. Otero (2011) concluye que aquellas empresas que se financiaron únicamente con fondos propios lograron menores niveles de crecimiento. Kantis (2009) encontró que uno de los factores de las empresas innovadoras dinámicas es tener diversas vías de financiamiento. Algunas empresas aceleran su crecimiento a partir de la utilización de fuentes externas de financiación provenientes de instituciones tales como bancos, organismos públicos o sociedades de capital riesgo (Guallarte, 2008). Diversos estudios han buscado explicar las causas que determinan que las empresas que obtuvieron financiación a través de fuentes externas, en particular de capital de riesgo, muestran mayor crecimiento. En la búsqueda de la explicación de esa relación se encuentra que, para acceder al capital de riesgo, las empresas pasan por un proceso de evaluación exigente (Gorman y Sahlman, 1989; Fried y Hisrich, 1994). Por otra parte, los inversionistas seleccionan a aquellas empresas que presentan una mayor probabilidad de crecimiento (Zacharakis y Meyer, 1998) y, finalmente, que el capital de riesgo no es sólo acce- so a recursos, sino también contactos (Davila, Foster y Gupta, 2003).

Otros estudios han verificado que aquellas empresas orientadas hacia la comercialización en mercados externos muestran, generalmente, tasas de crecimiento más altas (Beck, Demirgüç-Kunt y Maksimovic, 2002). Las empresas para acceder a mercados internacionales tienen que enfrentarse a barreras de entrada importantes, además, enfrentan una mayor competencia lo que les lleva a desarrollar una ventaja competitiva fuerte y, en general, tienen una mayor probabilidad de obtener ganancias importantes (Acs, Morck, Shaver y Yeung, 1997). Hessels y Van Stel (2011) establecen que las empresas exportadoras tienen mejores niveles de desempeño a través de una acumulación de recursos y capacidades.

Otro factor analizado es la rapidez en el proceso de la creación de una nueva empresa. Pues, cuando la creación de una empresa es fruto de un estudio adecuado y sistemático, esta tiene más probabilidades de crecer más rápidamente (Capelleres y Kantis, 2009).

Los equipos emprendedores son la suma de los recursos y las capacidades de sus miem- bros (Westhead y Cowling, 1995). Por eso, otros de los determinantes del crecimiento de las empresas son las capacidades del equipo emprendedor. En los últimos años se ha desarrollado una línea dedicada al estudio de los equipos de investigación (Ucbasaran, Lockett, Wright, y Westhead, 2003; Chandler, Honig y Wiklund, 2005). Las empresas fundadas por más de un individuo suelen tener mejores perspectivas de crecimiento en la medida en que son integradas por un equipo armónico y complementario entre sí (Harper, 2008). La existencia de un equipo emprendedor permite acceder a una mayor variedad de recursos, desarrollar más capacidades, compartir riesgos y responsabilidades que llevan a fijarse objetivos más ambiciosos (Terjesen y Szerb, 2008; Gilbert, McDougall y Audretsch, 2006).

Según el estudio realizado por Capelleres y Kantis (2009), en Chile las empresas innovadoras y dinámicas se caracterizan por introducir cambios organizacionales y por la revisión y ajuste de su modelo de negocios.

\section{Técnicas de análisis}

Existen diferentes técnicas de análisis utilizadas en estudios empíricos para $\gg$

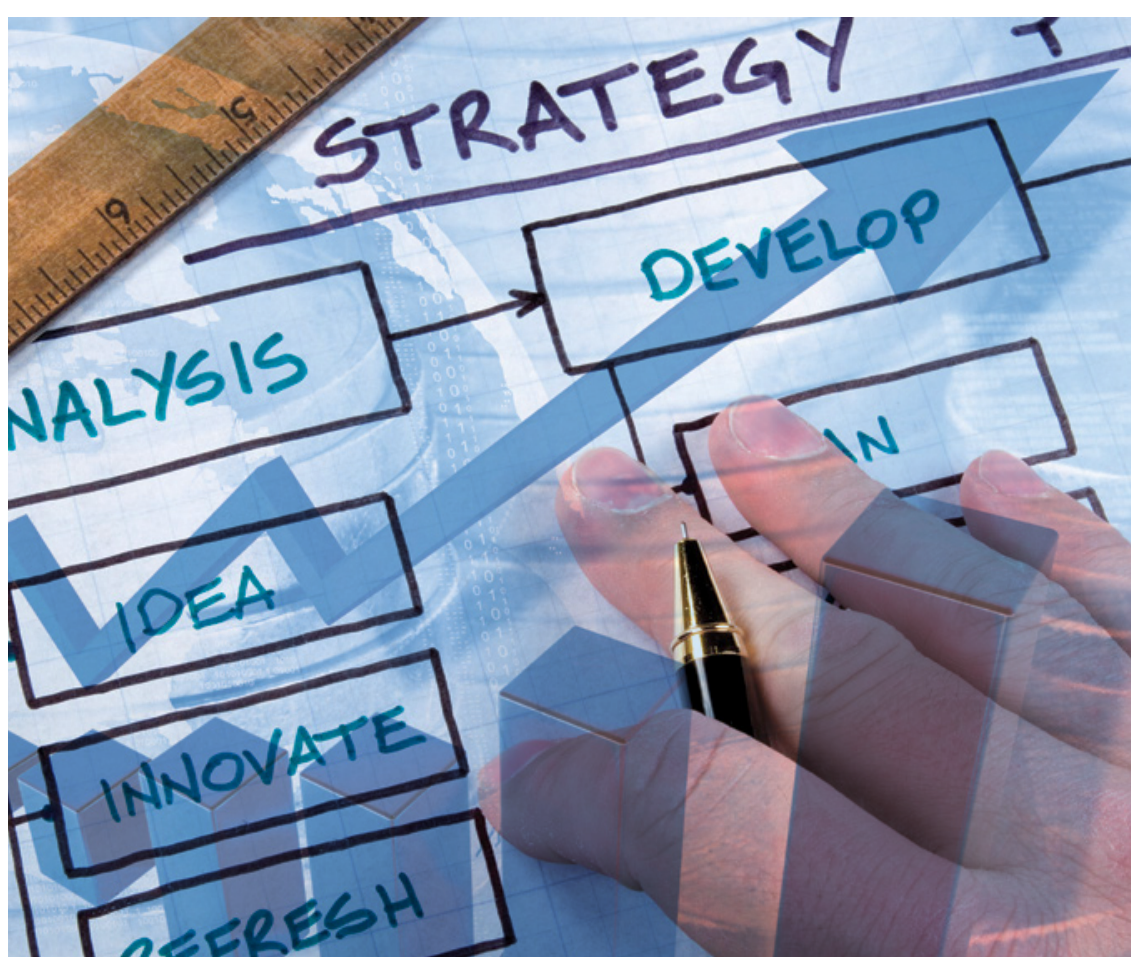


$\gg$ la identificación de factores de éxito. A continuación se presenta un relevamiento de las técnicas de análisis más importantes.

Utilizando el test de significancia Chi-cuadrado, Bonilla y Bermúdez (2013) analizan las diferencias por género en el emprendimiento empresarial. Así, determinan que la participación de mujeres es restringida y que la mujer emprende con mayor riesgo, menor experiencia y menor conocimiento empresarial. Además, existe una notoria brecha de ingresos y desigualdad de oportunidades.

Con el objetivo de identificar las principales variables que afectan las diversas etapas por las cuales atraviesa un emprendimiento y desarrollar un marco teórico-analítico, Aguirre (2011) desarrolla modelos ordinales logísticos, con variables dependientes no estandarizadas e independientes estandarizadas. Asimismo, identifica cuatro variables que acompañan todo el proceso.

Con base en técnicas de data mining, Martens, Vanhoutte, De Winne, Baesens, Sels y Mues (2011) se proponen identificar configuraciones de recursos iniciales, estrategia y medioambiente que conducen a un desempeño superior de start-ups. Con base en la técnica de árboles de decisión, identifican cinco configuraciones asociadas a ventajas competitivas y ocho con desventajas competitivas.

Mediante el análisis factorial y regresión múltiple, Entrialgo, Fernández y Vázquez (2010) analizan las características del comportamiento emprendedor y su vínculo con la estrategia, el entorno industrial y el éxito de la empresa. Por medio del análisis factorial, caracterizan el comportamiento emprendedor; y por medio de regresión múltiple, analizan el poder predictivo de diversos elementos sobre el éxito.

Con el objetivo de modelar la relación entre restricciones financieras y el éxito de startups, Stucki (2013) estudia la evolución de una cohorte de empresas y realiza estimaciones a partir de modelos probit multinomiales. En su estudio, el éxito se evalúa de dos formas: la sobrevivencia de la firma y el logro de beneficios, por tanto construyen modelos considerando dos variables dependientes.

Para determinar los factores que influ-
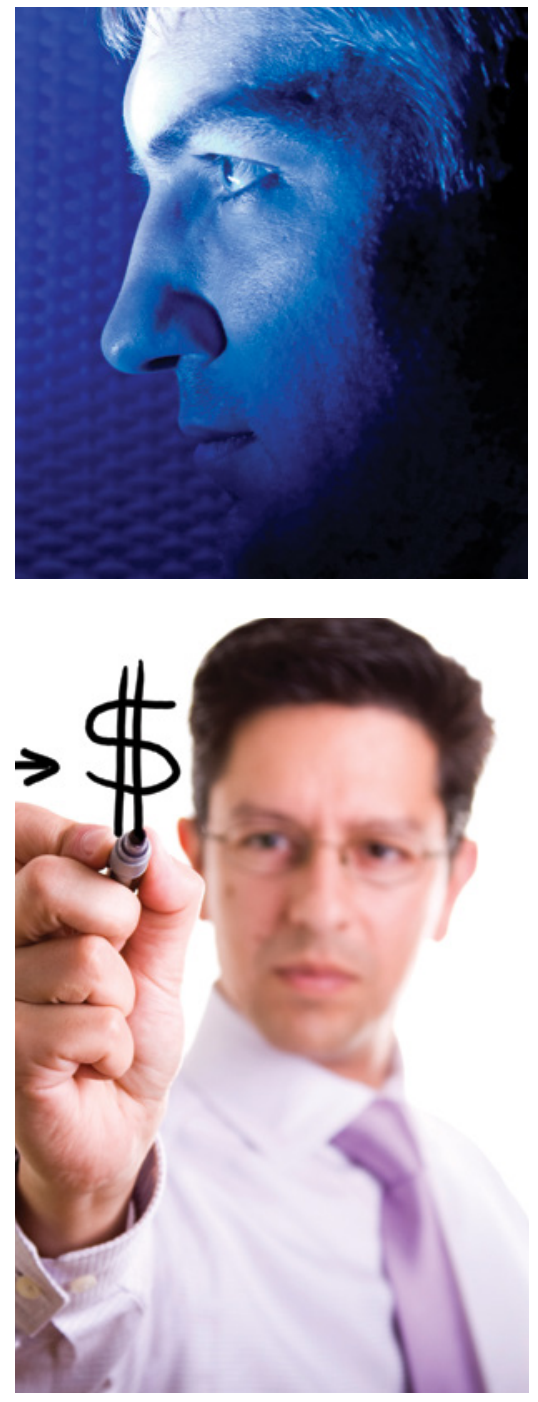

yen en el emprendimiento de los inmigrantes sudamericanos en Chile, Mancilla y De Lima (2014) aplican un modelo logístico para determinar el impacto sobre la probabilidad de emprendimiento individual. Determinan que el tiempo de residencia, la forma legal de ingreso al país, el nivel educacional y la experiencia previa en emprendimientos influyen en la probabilidad de crear una actividad empresarial.

\section{METODOLOGÍA}

Este trabajo se propone indagar y definir cuáles son los factores o características personales y del contexto que determinan el éxito de un emprendedor. Luego de analizar la po- tencialidad de las diferentes técnicas de análisis, se resolvió aplicar técnicas de minería de datos (data mining) (Piatetsky-Shapiro y Fayyad, 2011), cuya esencia radica en encontrar conocimiento nuevo y útil en los datos. Existe una gran diversidad de técnicas de minería de datos, algunas de las cuales tienen origen en la estadística multivariada y en las series temporales. Para lograr el objetivo propuesto se pueden utilizar una amplia gama de técnicas, además de las presentadas en este artículo, tales como redes neuronales y chi cuadrado bietápico, entre otras.

Se aplican dos técnicas de clasificación: árbol de decisión y regresión logística. Los resultados de ambos análisis son coincidentes y muestran que los dos elementos más relevantes para el éxito de un emprendimiento son contar con financiamiento y la situación laboral previa del emprendedor.

Los datos analizados provienen de una encuesta realizada a todos los participantes de los cursos del Programa de Emprendedores CCEEmprende. Los emprendedores considerados participaron en CCEEmprende entre los años 2007 y 2010, y se incluyeron sólo los 63 que culminaron el plan de negocios.

La encuesta abarca los siguientes aspectos: características sociodemográficas del emprendedor (edad, sexo, nivel de estudios, situación laboral); motivos para iniciar el emprendimiento (diferentes tipos de apoyo: familiar, económico, moral); motivos para asistir al programa CCEmprende; si se cuenta con financiamiento para el proyecto; e indicadores de éxito (si el proyecto logró constituirse/si genera ingresos). Como se mencionó anteriormente, en este trabajo se considera exitoso a un emprendimiento si se logra crear una empresa.

\section{RESULTADOS}

\section{Caracterización de los encuestados}

A continuación se presenta la caracterización de los emprendedores que participan del estudio. La edad promedio al ingresar al programa es poco más de 30 años, la mediana es 27 años (lo que significa que por lo menos el 


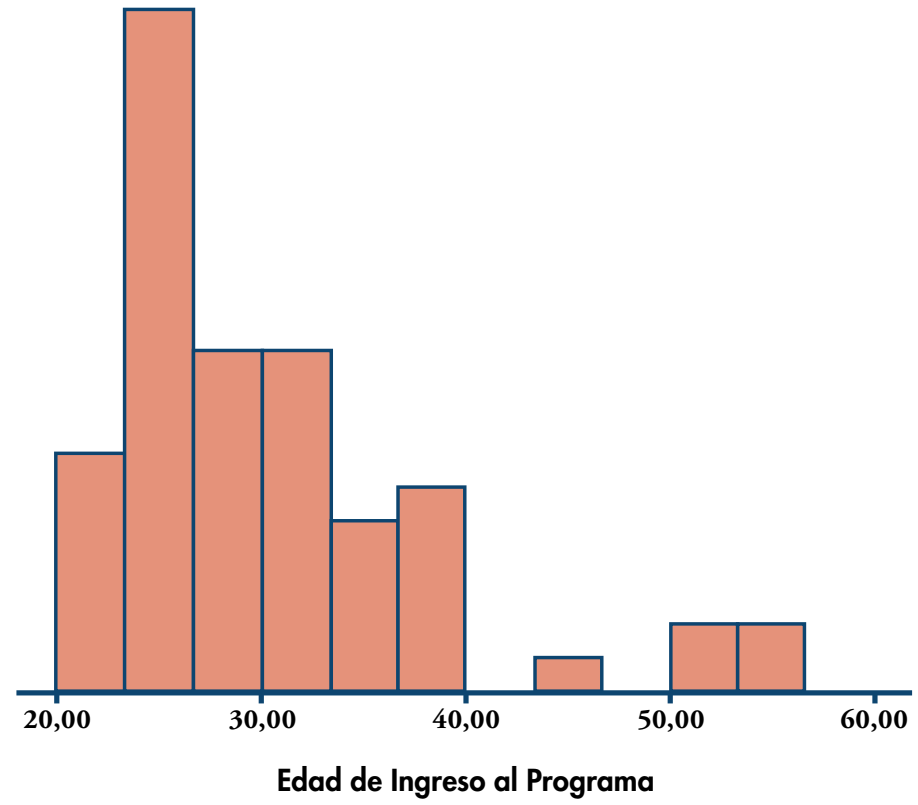

Figura 2: Histograma de la edad de ingreso al programa

$50 \%$ tiene 27 años o menos, y como máximo el 50\% tiene más de 27 años). El coeficiente de variación $(0,27)$ muestra una variabilidad razonable (véase figura 2). Son relativamente “jóvenes” en comparación a la población del país y tienen un nivel educativo principalmente universitario (figura 3). Además, debe tenerse en cuenta que el perfil está asociado al público que está generalmente más vinculado a los proyectos universitarios. En cuanto al sexo de los participantes: el $41 \%$ son mujeres y el $59 \%$ son hombres (figura 4 ).

\section{Aplicación de los modelos de clasificación}

La importancia de poder anticipar el éxito resulta fundamental para poder asignar adecuadamente los recursos disponibles en cuanto a capacitación y ayuda a los emprendedores, además para contar con una aproximación del riesgo de los proyectos. Se utilizaron dos técnicas de clasificación: árbol de decisión y la regresión logística. La variable dependiente definida fue "emprendimiento constituido" (con las categorías: Sí/No), que indica la creación (o no) de una empresa. Se consideraron las siguientes variables independientes: edad al ingreso al programa, nivel de estudios, tipo de estudios, situación laboral al ingresar al programa, motivo principal para emprender, participación anterior en otros programas de apoyo a emprendimientos, si se tiene financiamiento, si se cuenta con apoyo familiar (económico, moral y otro tipo). Con base en los análisis efectuados, se identifica cuáles de las variables independientes tienen incidencia significativa en la creación de la empresa.

Utilizando árboles de decisión, se obtuvo que los dos factores relevantes para anticipar si la empresa se creará son: a) conseguir financiamiento ${ }^{2}$ y b) la situación laboral preexistente del emprendedor (como se muestra en la figura 5) considerando un nivel de significación del 5\%. Al analizar el árbol de decisión, se observa que en la raíz los porcentajes de Sí y No son similares. En el segundo nivel, al subdividir en función de tener o no financiamiento, el porcentaje de Sí es consi-

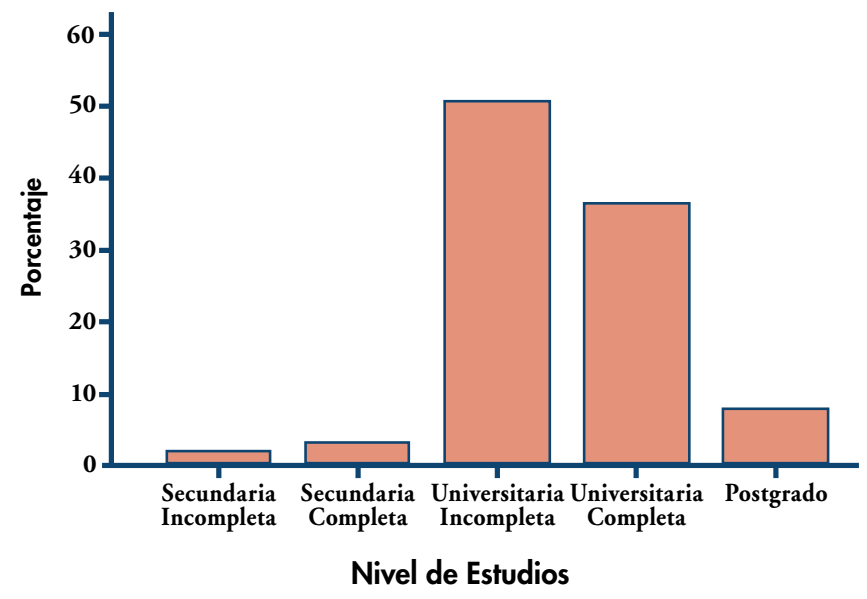

Figura 3: Distribución del nivel de estudios

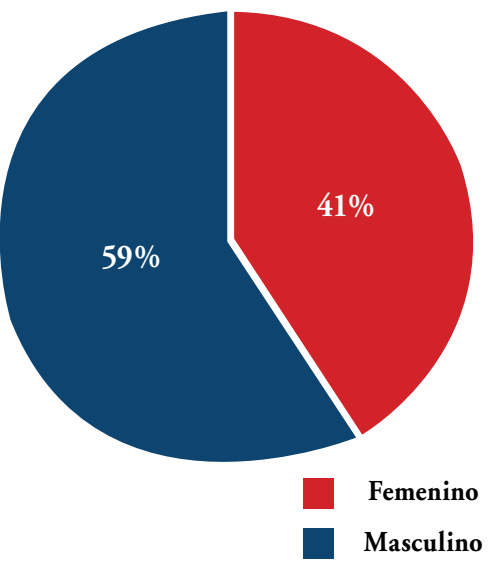

Figura 4: Distribución por sexo de los participantes en el programa 
$\gg$ derablemente mayor para quienes tienen financiamiento, y el porcentaje de No es superior para quienes no tienen financiamiento. Por último, en el tercer nivel se pueden apreciar dos rutas relevantes: tener financiamiento y actividad laboral independiente muestra un alto porcentaje de emprendimientos consti- tuidos; en cambio no tener financiamiento y no tener actividad laboral independiente (puede ser dependiente o no trabajar) muestra un alto porcentaje de emprendimientos no constituidos. De esta forma quedan identificadas la "ruta del éxito" y la "ruta del fracaso".

\section{EMPRENDIMIENTO CONSTITUIDO}
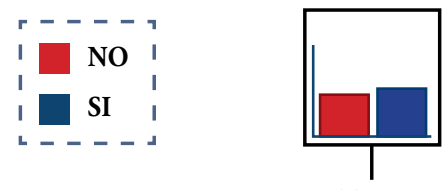

Tiene

Financiamiento

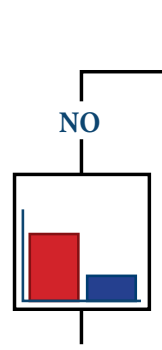

$$
\text { Situación }
$$

Laboral

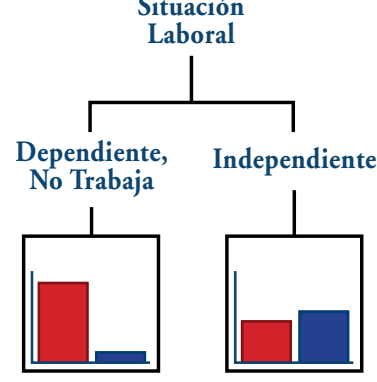

Figura 5: Ruta del éxito

Tabla 1: Regresión logística

\begin{tabular}{|c|c|c|c|c|c|c|}
\hline & B & E.T. & Wald & Gil & Sig. & Exp.(B) \\
\hline Situación Laboral & 1,91442243 & 0,69882531 & 7,50478564 & 1 & 0,00615353 & 6,78302 \\
\hline $\begin{array}{c}\text { Tiene } \\
\text { Financiamiento }\end{array}$ & 2,29156883 & 0,75901763 & 9,11511369 & 1 & 0,00253506 & 9,89044196 \\
\hline \begin{tabular}{c} 
Constante \\
\hline
\end{tabular} & $-4,05342746$ & 1,3272385 & 9,32710504 & 1 & 0,00225789 & 0,01736276 \\
\hline & a Variable(s) introducida(s) en el paso 1: Tiene Financiamiento. \\
\hline & b Variable(s) introducida(s) en el paso 2: Situación Laboral. \\
\hline
\end{tabular}

Por otra parte, con base en la regresión logística (incluyendo variables por el método Wald), se encuentra que la variable más relevante (que se incluye en el primer paso) es tener financiamiento, y la segunda en importancia (que se agrega en el segundo paso) es la situación laboral preexistente del emprendedor. El modelo final obtenido se presenta en la tabla 1.

Como resultado, se observa que ambos métodos muestran las mismas variables relevantes y en el mismo orden.

\section{CONCLUSIONES Y TRABAJOS FUTUROS}

Poder anticipar el éxito de un proyecto resulta de gran interés para identificar emprendimientos con potencial y las áreas en las cuales brindar apoyo. Los hallazgos que presenta el estudio de caso presentado aportan información potencialmente útil para la toma de decisiones, relativa a cómo canalizar el apoyo a emprendedores, en particular, en cuanto a cuáles son las formas de apoyo más efectivas, las mejores alternativas para crear incentivos al emprendedor y conocer las características que deben tener las herramientas o actividades que más contribuyen al éxito de los emprendimientos. El trabajo presentado constituye el primer estudio realizado en Uruguay en la temática, si bien desde la teoría o para otras realidades existe información sobre los factores que colaboran en la determinación del éxito.

Estos resultados muestran la importancia del financiamiento a emprendedores y de la situación laboral preexistente del emprendedor. A partir de ahí se podría ahondar en el contexto familiar y socioeconómico (tanto por su impacto en la intención de emprender como por la situación económica familiar), y en el estudio de las posibilidades de financiamiento existentes.

Si bien los resultados de las dos técnicas aplicadas son coincidentes, es importante destacar que, 


\section{Los hallazgos muestran que los dos elementos más relevantes para anticipar el éxito de un emprendimiento son contar con financiamiento y que, anteriormente, la situación laboral del emprendedor sea trabajador independiente}

para quienes están en contacto permanente con los emprendedores, les resulta más sencillo de visualizar el análisis a partir de árboles de decisión.

Este trabajo presenta algunas limitaciones, asociadas a variables no relevadas en la encuesta disponible y a que solamente se cuenta con $63 \operatorname{casos}^{3}$ (que, sin embargo, representan adecuadamente a la población objetivo). Constituye un estudio basado en métodos cuantitativos de investigación, lo que deja por fuera los hallazgos que podrían obtenerse al utilizar metodología cualitativa, tales como la historia detallada del proceso emprendedor, motivaciones, sentimientos, entre otros.

A partir de los resultados de este trabajo, se abren nuevas posibilidades de extender el estudio en varios sentidos: considerar otras formas de entender el éxito de un proyecto; la aplicación de otras técnicas de data mining, incluyendo métodos cualitativos de investigación; utilizar más variables y extenderlo a otros conjuntos de emprendedores y emprendimientos. Asimismo, se está desarrollando un Data Warehouse, con el objetivo de contar con un modelo multidimensional que permita realizar consultas OLAP (On Line Analytical Processing) por parte de diversos actores.

\section{Referencias Bibliográficas}

Acs, Z., Morck, R., Shaver, J. \& Yeung, B. 1997. The internationalization of small and medium-sized enterprises: a policy perspective. Small Business Economics, 9(1), 7-20.

Aguirre, J. A. (2011). La transformación emprendedora en estudiantes universitarios en el contexto de Costa Rica: del interés por, a sentirse listo para iniciar cosas. Memorias XXI Congreso
Latinoamericano Sobre Espiritu Empresarial. Universidad Icesi. Colombia.

Alemany, L., Álvarez, C., Planellas, M y Urbano, D. (2011): Libro blanco de la cultura emprendedora en España. Fundación Príncipe de Girona. ESADE. Barcelona. Disponible en: http://www. cise.es/wp-content/uploads/2013/03/9_LBIEE_ Documento-Final.pdf

Almus, M. (2002). What characterizes a fast-growing firm?. Applied Economics, 34, $1497-$ 1508.

Arribas, I. y Vila, J. (2004). La actitud emprendedora del universitario valenciano. El emprendedor innovador y la creación de empresas de I+ D+ I. Valencia: PUV.

Beck, T., Demirgüç-Kunt, A. \& Maksimovic, V. 2002. Financial and legal constraints to firm growth: Does size matter? Policy Research Working Paper 2784.The World Bank Research Group (Finance).

Bonilla, L. y Bermúdez, L. (2013). Diferencias por género en el emprendimiento empresarial costarricense. Tec Empresarial, 7(2), 19-27.

Brüderl, J. y Preisendörfer, P. (2000). Fast-growing businesses: empirical evidence from a German study. International Journal of Sociology, 30(3), 45-70.

Capelleras, J. y Greene, F. (2008). The determinants and growth implications of venture creation speed. Entrepreneurship and Regional Development, 20(4), 317-343.

Capelleras, J. y Kantis, H. (2009). Nuevas empresas en América Latina: factores que favorecen su rápido crecimiento. Universidad Autónoma de Barcelona. España.
Chandler, G., Honig, B. \& Wiklund, J. (2005). Antecedents, moderators, and performance consequences of membership change in new venture teams. Journal of Business Venturing, 20(5), 705-25.

Colombo, M. \& Grilli, L. (2005). Founder's human capital and the growth of new technology-based firms: A competence-based view. Research Policy, 34, 795-816.

Davidsson, P., Delmar, F. \& Wiklund, J. (2006), Entrepreneurship and the Growth of Firms. Cheltelham: Elgar.

Davila, A., Foster, G. \& Gupta, M. (2003). Venture capital financing and the growth of startup firms. Journal of Business Venturing, 18(6), 689-708.

De Castro, J., Pistrui, J., Coduras, A., Cohen, B. y Justo, R. (2002). Proyecto GEM: informe ejecutivo 2001. Cátedra Najeti-Instituto de Empresa, Madrid.

Entrialgo, M., Fernández, E. y Vázquez, C. (2010). El comportamiento emprendedor y el éxito de la PYME: modelos de contingencia y configuracionales. Revista Dirección y Organización, 25, 47-58.

Evans, D. S., \& Leighton, L. S. (1989). Some empirical aspects of entrepreneurship. The American Economic Review, 79(3), 519-535.

Fernández, E., Montes, J. y Vázquez, C. (2010). Los recursos intangibles como factores de competitividad de la empresa. Revista Dirección y Organización, 20, 84-98.

Fried, V. y Hisrich, R. (1994). Toward a model of venture capital investment decision making. Financial management, 28-37.

García, P. y Jiménez, A. (2012). Emprendedores y empresas. La construcción social del em- 》 
$\gg$ prendedor. Lan Harremanak revista de Relaciones Laborales, 24, 219-236.

Gilbert, B., McDougall, P. \& Audretsch, D. (2006). New venture growth: A review and extension. Journal of Management, 32(6), 926-950. Crecimiento y adaptación en un contexto de crisis internacional: los casos de tres empresas jóvenes dinámicas argentinas. Programa de Desarrollo Emprendedor (Prodem). Instituto de Industria (IDEI), Universidad Nacional de General Sarmiento (UNGS), Instituto de Industria (IDEI). (inédito).

Gorman, M. \& Sahlman, W. (1989). What do venture capitalists do?. Journal of Business Venturing, 4(4), 231-248.

Guallarte, C. (dir.) (2008). Informe Ejecutivo Cataluña. Global Entrepreneurship Monitor.

Harper, D. (2008). Towards a theory of entrepreneurial teams. Journal of Business Venturing, 23(6), 613-626.

Hessels, J. \& Van Stel, A. (2011). Entrepreneurship, export orientation, and economic growth. Small Business Economics, 37(2), 255-268.

Honig, B. (2001). Human capital and structural upheaval: A study of manufacturing firms in the
Gonzalo, M., Federico, J. y Kantis, H. (2013)

West Bank. Journal of Business Venturing, 16(6), 575-594.

Kantis, H., Federico, J. y Menéndez, C. (2012). Políticas de fomento al emprendimiento dinámico en América Latina: tendencias y desafíos. CAF Documentos de Trabajo N ${ }^{\circ}$ 2012/09.

Kantis, H., Angelelli, P. y Moori-koenig, V. (2004). Desarrollo Emprendedor. América Latina y el Contexto Internacional. Editorial Nomos (Colombia) Editorial Temas (Argentina).

Lafuente, E., Vaillant, Y. y Gómez, E. (2011). El impacto de factores socio-culturales sobre la actividad emprendedora de los jóvenes en España. Universidad Icesi. Cali.

Leiva, J. (2013). ¿Quién crea MIPYMES en Costa Rica?. Tec Empresarial, 7(2), 9-17.

Mancilla, C y De Lima, P (2014). Nuevos inmigrantes en Chile: Los determinantes de su actividad emprendedora. Migraciones Internacionales. 7(4), 235-264. Disponible en: https://5dafb8fd88a2747021f681f1aa3329c504fcf20c.googledrive.com/host/0BwUFLG5ISAKvZjNIa001SzlwUTg/08-MI27-235-264.pdf

Martens, D., Vanhoutte, C., De Winne, S., Baesens, B., Sels, L., \& Mues, C. (2011). Identifying financially successful start-up profiles with data

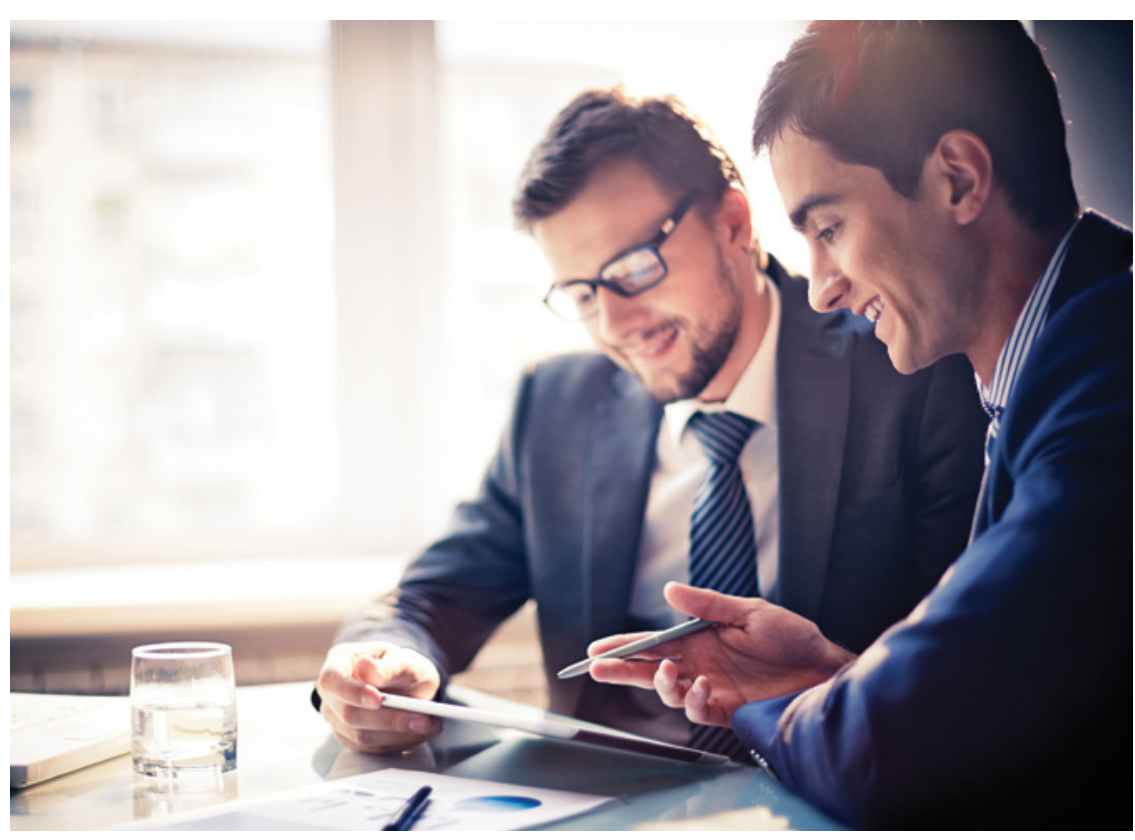

mining. Expert Systems with Applications, 38(5), 5794-5800.

Otero, A. (2011). Factores claves en el proceso de acceder a recursos financieros en Colombia para nuevos empresarios. Memorias XXI Congreso Latinoamericano sobre Espíritu Empresarial. Disponible en: http://bibliotecadigital.icesi.edu.co/ biblioteca_digital/bitstream/item/5387/1/7P8.pdf Piatetsky-Shapiro, G. \& Fayyad, U. (2011). An introduction to SIGKDD and a reflection on the term'data mining.' SIGKDD Explorations, 13(2), 102-103.

Sastre, R. (2013). La motivación emprendedora y los factores que contribuyen con el éxito del emprendimiento. Ciencias Administrativas. Revista Digital FCE, 1(1), 1-10.

Stucki, T. (2013). Success of start-up firms: the role of financial constraints. Industrial and Corporate Change. Oxford University Press.

Talín, J. y Cuesta, S. (2013). El emprendimiento y la innovación como fundamento de la creación de pequeñas y medianas empresas. Revista de Extensión Universitaria, 1(3), 48-55.

Terjesen, S. \& Szerb, L. (2008). Dice thrown from the beginning? An empirical investigation of determinants of firm level growth expectations. Estudios de Economía, 35(2), 154-178.

Ucbasaran, D., Lockett, A., Wright, M. \& Westhead, P. (2003). Entrepreneurial founder teams: Factors associated with member entry and exit. Entrepreneurship Theory and Practice, 28(2), 107-128.

Vaillant, Y. \& Lafuente, E. (2007). Do different institutional frameworks condition the influence of local fear of failure and entrepreneurial examples over entrepreneurial activity?. Entrepreneurship and Regional Development, 19(4), 313-337.

Westhead, P. \& Cowling, M. (1995). Employment change in independent owner-mana ged high-technology firms in Great Britain. Small Business Economics, 7(2), 111-140.

Zacharakis, A. \& Meyer, G. (1998). A lack of insight: do venture capitalists really understand their own decision process?. Journal of Business Venturing, 13(1), 57-76. 